Schmerz 2020 $34: 301-302$

https://doi.org/10.1007/s00482-020-00484-8

(c) Springer Medizin Verlag GmbH, ein Teil von Springer Nature 2020

Lukas Radbruch ${ }^{1,2} \cdot$ Hans-Georg Schaible ${ }^{3}$

${ }^{1}$ Klinik für Palliativmedizin, Universitätsklinikum Bonn, Bonn, Deutschland

${ }^{2}$ Zentrum für Palliativmedizin, Malteserkrankenhaus Bonn/Rhein-Sieg, Bonn, Deutschland

${ }^{3}$ Institut für Physiologie, Universität Jena, Jena, Deutschland

\title{
Isolieren, bis es weh tut - die Folgen der Corona-Pandemie
}

In den letzten Wochen und Monaten ist eine Fülle von Leitlinien und Empfehlungen zur Corona-Pandemie veröffentlicht worden. Über die Deutsche Gesellschaft für Palliativmedizin war ich (LR) zum Beispiel in die Entwicklung der Leitlinien zur Entscheidungsfindung bei knappen Ressourcen (federführend Deutsche Interdisziplinäre Vereinigung für Intensiv- und Notfallmedizin [DIVI] und Akademie für Ethik in der Medizin [AEM]; [1]), zur Symptomkontrolle bei COVID-19 (federführend Deutsche Gesellschaft für Palliativmedizin [DGP]; [2]) und zur ambulanten Versorgung (federführend die Deutsche interprofessionelle Vereinigung für Behandlung im Voraus Planen [DiV-BVP]; [3]) beteiligt.

In diesen Leitlinien wurde aber nur wenig auf die Folgen der sozialen Isolierung eingegangen, obwohl diese Folgen in der Öffentlichkeit wie auch im Alltag der klinischen Versorgung deutlich zu spüren sind. In erster Linie sind es natürlich die Patienten mit positivem Virennachweis, die unter dem Besuchsverbot auf der Isolierstation litten, bis hin zu den Berichten über Patienten mit COVID-19, die auf der Intensivstation versterben, ohne dass die Angehörigen sie noch einmal besuchen konnten.

Aber auch viele andere Menschen sind von den Isolierungs- und Distanzierungsmaßnahmen betroffen. Ambulante Hospizdienste haben die Besuche der ehrenamtlichen Mitarbeiter eingestellt, ebenso ist vielerorts Psychotherapie oder Seelsorge nur noch telefonisch möglich. Im Palliativdienst betreute Patienten brachen die palliative Chemotherapie ab, weil sie dafür im Krankenhaus isoliert worden wären und lieber die verbleiben- de Lebenszeit mit der Familie verbringen wollten. Der Ehemann einer Patientin konnte seine Frau nicht auf der Palliativstation besuchen, weil das Pflegeheim, in dem er lebte, ihm mitteilte, dass er nach einem solchen Besuch nicht mehr zurück ins Pflegeheim kommen dürfte. In der SAPV bricht der Ehemann der verstorbenen Patientin in Tränen aus, als er realisiert, dass er maximal 10 Menschen zur Beerdigung einladen kann. Auf der Palliativstation wird beim routinemäBigen Abstrich vor der Verlegung des Patienten ins Pflegeheim ein positiver Befund gemeldet, daraufhin müssen fast alle Ärzte und Pflegekräfte der Station in häusliche Quarantäne - sollen allerdings auch weiter mit Mundschutz arbeiten kommen, da sonst der Stationsbetrieb nicht aufrechterhalten werden kann.

Auch wenn jetzt nach und nach die Bestimmungen gelockert werden, Besuche im Pflegeheim oder Krankenhaus wieder möglich sind (wenn auch mit starken Einschränkungen), sieht es doch danach aus, dass die Corona-bedingten Probleme uns noch länger beschäftigen werden.

Dabei ist eigentlich trotz aller Einschränkungen vieles möglich. Das fängt übrigens schon bei der Terminologie an. Wir möchten nicht von sozialer Distanzierung reden, weil ja gerade die sozialen Beziehungen erhalten und möglichst noch gestärkt werden sollen - auch wenn man sich nicht berühren darf. Es sollte deshalb besser der Begriff der physischen Distanzierung genutzt werden.

Von den vielen Ideen lässt sich sicherlich nicht alles umsetzen. So hat einer der Autoren (LR) mehrere Tablets gekauft und für Patienten eingerichtet, die in der Isolierung mit ihren Angehörigen über 
soziale Medien kommunizieren möchten. Diese Tablets wurden bis jetzt aber noch nicht gebraucht, weil die betroffenen Patienten entweder körperlich und kognitiv so eingeschränkt waren, dass sie die Geräte nicht nutzen konnten oder, wenn sie dafür noch fit genug waren, ohnehin schon über ihr Smartphone alle Kontaktmöglichkeiten genutzt hatten.

Dennoch sind wir gefordert, neue Wege zu beschreiten, zum Beispiel mit Angeboten zur virtuellen Trauerbegleitung, wenn die realen Kontakte mit Freunden, Verwandten oder Trauerbegleitern nicht möglich sind. In den Empfehlungen der Deutschen Gesellschaft für Palliativmedizin und anderer Fachgesellschaften, die in dieser Ausgabe von Der Schmerz veröffentlicht werden [4], sind solche neuen Wege gesammelt worden. Dabei geht es um die betroffenen Patienten und ihre Angehörigen, aber auch um die Behandlungsteams auf den Isolier- und Intensivstationen, die die Last der COVID-19Behandlungen tragen müssen, und die Hospiz- und Palliativteams.

Vielleicht stellen wir ja im Lauf der Pandemie fest, dass sich mit den neuen Wegen auch neue Horizonte eröffnen, und manches auch nach der Pandemie noch genutzt werden kann. Nicht als Ersatz für Nähe oder Berührung, aber als Ergänzung, zum Beispiel mit niederschwelligen Angeboten für die Trauerbegleitung über soziale Medien.

Wir möchten Sie weiterhin anhalten, Ihre Erfahrungen und Erkenntnisse aus der Corona-Pandemie mit den Lesern von Der Schmerz zu teilen, wenn sie auch für andere Behandler relevant sind [5]!

\section{Korrespondenzadresse}

Prof. Dr. Lukas Radbruch

Klinik für Palliativmedizin, Universitätsklinikum Bonn

Sigmund-Freud-Str. 25, 53127 Bonn,

Deutschland

Lukas.Radbruch@malteser.org

Interessenkonflikt. L. Radbruch und H.-G. Schaible geben an, dass kein Interessenkonflikt besteht.

\section{Literatur}

1. Deutsche Interdisziplinäre Vereinigung für Intensiv- und Notfallmedizin, Deutsche Gesellschaft für Interdisziplinäre Notfall- und Akutmedizin, Deutsche Gesellschaft für Anästhesiologie und Intensivmedizin, Deutsche Gesellschaft für Internistische Intensivmedizin und Notfallmedizin, Deutsche Gesellschaft für Pneumologie und Beatmungsmedizin, Deutsche Gesellschaft für Palliativmedizin, Akademie für Ethik in der Medizin (2020) Entscheidungen über die Zuteilung von Ressourcen in der Notfall- und der Intensivmedizin im Kontext der Covid-19-Pandemie: Klinisch ethische Empfehlungen. https://www.divi.de/ empfehlungen/publikationen/covid-19/1540covid-19-ethik-empfehlung-v2/file. Zugegriffen: 10.Apr. 2020

2. Nehls W, Delis S, Haberland B, Maier BO, Sanger K, Tessmer G, Radbruch L, Bausewein C (2020) Therapie von PatientInnen mit COVID19 - Empfehlungen aus der Perspektive der Palliativversorgung. Pneumologie. https://doi. org/10.1055/a-1156-2759

3. Deutsche interprofessionelle Vereinigung - Behandlung im Voraus Planen, Deutsche Gesellschaft für Allgemeinmedizin, Deutsche Gesellschaft für Palliativmedizin, Deutsche Gesellschaft für Pneumologie und Beatmungsmedizin, Deutsche Gesellschaft für Anästhesiologie und Intensivmedizin, Deutsche Interdisziplinären Vereinigung fü Intensiv- und Notfallmedizin (2020) Ambulante patienten-zentrierte Vorausplanung für den Notfall - Ein Leitfaden aus Anlass der Covid19-Pandemie. https://www.dgpalliativmedizin. de/images/Ambulante_patientenzentrierte Vorausplanung_fuer_den_Notfall_LEITFADEN_ 20200409_final.pdf.Zugegriffen: 15. Mai 2020

4. Münch U, Müller H, Deffner T et al (2020) Empfehlungen zur Unterstützung von belasteten, schwerstkranken, sterbenden und trauernden Menschen in der Corona-Pandemie aus palliativmedizinischer Perspektive. Schmerz. https://doi. org/10.1007/s00482-020-00483-9

5. Radbruch L, Schaible HG (2020) COVID-19 und Schmerz - Call for Papers. Schmerz. https://doi. org/10.1007/s00482-020-00475-9
Schmerz - eine Herausforderung

Ein Ratgeber für Betroffene und Angehörige

3., vollst. akt. u. erw. Aufl. 2020, XVII, 204S. 52 Abb.

Hans-Günter Nobis · Roman Rolke

Toni Graf-Baumann Hisg.

Schmerz-eine

Herausforderung

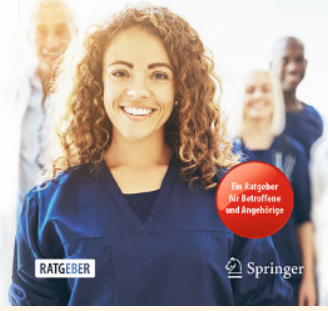

- Umfangreiche Informationen von führenden Schmerzexperten: aktuell, neutral und wissenschaftlich fundiert

- Empfohlen von der Deutschen Schmerzgesellschaft e.V., der Deutschen Gesellschaft für Psychologische Schmerztherapie und forschung sowie der Deutschen Migräne- und Kopfschmerzgesellschaft

Bei mehr als der Hälfte aller Menschen mit chronischem Schmerz dauert es mehr als 2 Jahre, bis sie eine wirksame Schmerzbehandlung erhalten. Dieses Buch hilft Betroffenen, sich gezielter professionelle Unterstützung zu suchen. Knapp 60 führende Schmerzexperten haben mit über 77 Beiträgen daran mitgewirkt. Betroffene und Angehörige erfahren mehr über die körperlichen, psychischen und sozialen Zusammenhänge von Schmerz, um die Behandlung motiviert und eigenverantwortlich mitzugestalten. So versteht der Betroffene die bio-psycho-sozialen Zusammenhänge von Schmerz aus Sicht der aktuellen Schmerzmedizin und -psychologie. Die 3. Auflage wurde komplett aktualisiert und umfassend erweitert, u.a. um die Themen "Schmerz und Sexualität", "Wachstumsschmerzen bei Kindern", „Endometriose“ und „Gelenkschmerz", „Naturheilkunde bei Schmerz" und was bei der Einnahme von Schmerzmitteln während der Schwangerschaft, Stillzeit, auf Reisen und beim Sport zu beachten ist. 